\title{
SULLA NATURA FISICA DEI MICROSISMI
}

\author{
G. D'HENRY
}

1. Premessa. - 11 problema dei microsismi ha assunto in questi ultimi tempi grande importanza; dal punto di vista pratico sarebbe notevolmente utile potere ricavare dalla registrazione dei microsimi in una sola stazione, la direzione di provenienza di questi e quindi, essendo ormai accertała la dipendenza dei microsismi dai cicloni (1) ( $\left.{ }^{2}\right)$, localizzare anche la posizione di questi ed i loro eventuali spostamenti.

In questo lavoro si cerca appunto se sia possibile stabilire la direzione di provenienza delle onde dallo studio delle componenti la perturbazione periodica secondo un sistema di assi ortogonali $x$, $y, z$, quali ci vengono fornite direttamente dai sismografi. Per questo si sono cercati alcuni microsismi originati da cicloni bene localizzati, in particolare con l'azimut secondo una delle componenti strumentali; e si è studiato il comportamento delle loro oscillazioni, con i metodi che ora esporremo. Ciò è stato fatto per i casi tipici più sotto specificati, sui fotosismogrammi di Trieste, dove però manca la componente verticale, e su quelli di Roma gentilmente concessi in prestito.

2. Metodo di Bungers. - Prima di entrare nel vivo dell'argomento si è tentato di applicare il metodo di Bungers. Il Bungers $\left({ }^{3}\right)$ aveva elaborato il seguente metodo per ricavare la direzione di provenienza dei microsismi: nell'ipotesi che essi risultassero dalla sovrapposizione di due treni di onde di Rayleigh con ampiezza pressocché costante e direzioni di provenienza differenti, si cercano sulle due componenti orizzontali due massimi in concordanza di fase, e la concordanza immediatamente successiva. Le ampiezze di questi massimi determinano i vertici di un parallelogramma, il cui lato maggiore è una delle direzioni di provenienza, il lato minore l'altra. Applicato ad alcuni casi, questo metodo ha dato però risultato negativo.

Avendo già constatata preliminarmente l'inapplicabilità del metodo, ci si è limitati a tre soli casi, ricavati da un diagramma particolarmente significativo regristrato a Trieste il 2] gennaio 1937; essi si riferiscono alle ore 4,55 alle 5,00 ed alle 5,02. In tale giorno un'area 
depressionaria è situata presso le coste orientali dell'Inglilterra cioè a NW della stazione registratrice (Trieste). Applicando il metodo di Bungers in relazione alle ampiezze registrate dai sismograf, le parallele ai lati del parallelogramma passanti per l'origine non indicano la direzione di provenienza, cioè $N \mathbb{W}$, bensì rispettivamente $W, W S W$, $\mathrm{W}$ ( fig. 1 ). $\hat{\mathrm{E}}$ invece nella direzione di provenienza la diagonale maggiore.

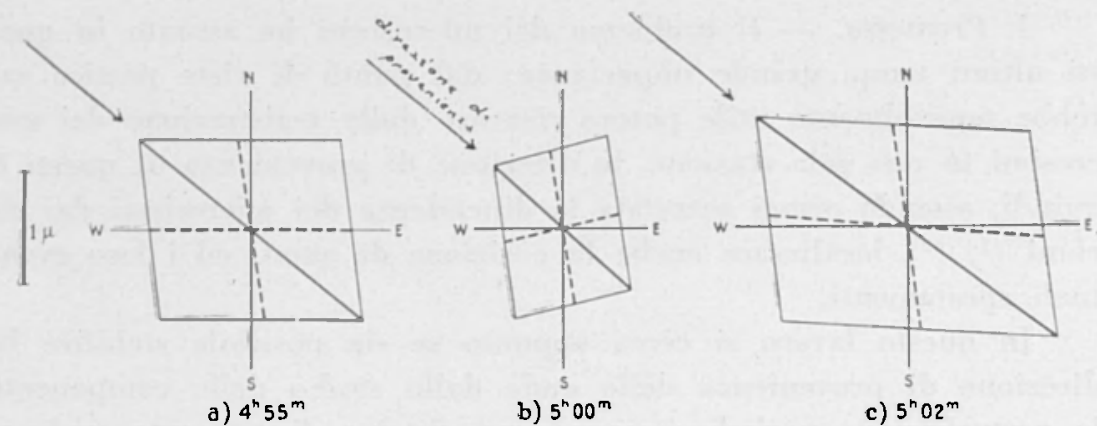

Fig. 1 - Applicazione del metodo di Bungers (21 gennaio 1937)

Dati questi risultati si può dedurre che ciò potrebhe dipendere dal fatto che, mentre il metodo di Bungers è stato elaborato per onde di Rayleiglı pure, i microsismi pare non abbiano questa caratteristica, ma presentino analogie con le onde massime dei terremoti lontani. Infatti secondo un recente studio $\left({ }^{4}\right)$, risulta che queste, ritenute finora esclusivamente polarizzate nel piano principale, si presentano invece anche con una spiccata componente trasversale.

3. Studio di microsismi dovuti a cicloni in azimut favorevole. Vista l'inapplicabilità del metodo di Bungers si sono allora cercati, fra le registrazioni di varie annate, esempi di cicloni o fronti fredli (avendo già constatato $\left({ }^{2}\right)$ la dipendenza dei microsismi pure dai fronti freddi) orientati nella direzione di una delle due componenti orizzontali (N-S o E-W). Fra questi, tutti quelli che presentavano qualche ambiguità riguardo la localizzazione del ciclone sono stati successivamente scartati; i casi disponibili rimasti non sono stati quindi molti, ma pure hanno offerto alcune interessanti considerazioni.

Esempi di cicloni o fronti freddi orientati in modo da dare registrazione (pre. dominante) su una sola componente, come se fossero composti prevalentemente da onde trasversali.

- Il 18 novembre 1936 un minimo è situato sull'Alto Adriatico. La compo- 
nente $N$ a Trieste non segnala nulla, la componente $E$ segnala tracce. dato il piccolo gradiente del ciclone, ma chiaramente visibili.

- Il 23.24 novembre 1940 un minimo è situato sull'Adriatico centrale in direzione S-SE rispetto a Trieste. I microsismi registrati dalla componente $\mathrm{E}$ ( fig. 2) sono più ampi di quelli registrati dalla componente $N$ (in proporzione ben maggiore di quanto dovuto alla diversità d'ingrandimento dinamirn N.S $=1400$. F.W $=170$ ก

- 116 dicembre 1940 un minimo $(755 \mathrm{mb})$ è situato sull'Adriatico centrale. La componente $\mathrm{E}-\mathrm{W}$ registra microsismi di carattere mediterraneo, mentre la componente N.S registra tracce ma anche queste molto rare.

- Nei giorni 16-17-18

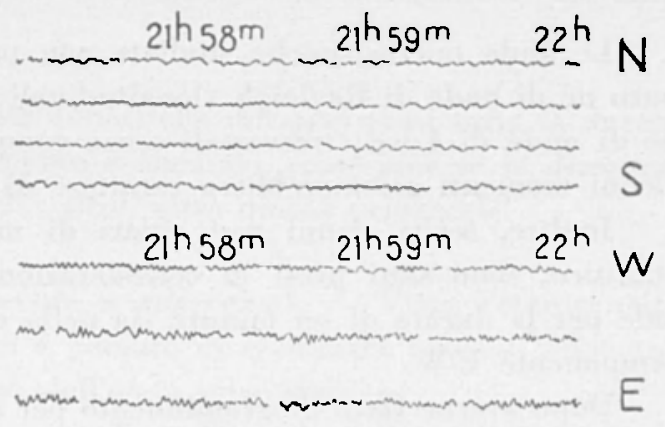
maggio 1948 un minimo si estende ad $W$ della Spagna, mantenendo costante la sua posizione per tutti e tre $i$ giorni. La perturbazione che ne deriva è registrata a Roma

Fig. 2 - Caso tipico di polarizzazione trasversale (23.2. novembre 1940)

solo dalla componente N.S, mentre la componente E.W, durante tutti i tre giorni, non registra alcun turbamento microsismico.

- Il 19 maggio alle $18^{\mathrm{h}}$ la situazione si inverte: cioc̀, mentre il ciclone di cui sopra è sulla Spagna, un altro minimo si forma a $N$ della costa norvegese. Questo ciclone viene registrato a Roma solo dalla componente E-W. La registrazione rimane tale per tutta la notte seguente.

- Il 19 maggio 1948 alle ore 1 un fronte freddo si trova nell'Adriatico centrale e settentrionale. Esso è di breve durata e non viene segnalato da microsismi (componente E-W) nella stazione di Trieste. A Roma la stazione segnala microsismi di periodo piccolo solo sulla componente N-S.

Ho messo quindi in relazione $\mathrm{i}$ bollettini meteorologici con i sismogrammi provvisti pure della componente verticale. Dopo avere scartato anche ora i casi di ambigua localizzazione del ciclone, sono rimasti disponibili i seguenti casi :

- Il lu gennaio 1949 un minimo si estende sull'Adriatico settentrionale e sull'Emilia, cioè a Nord della stazione di Roma; ma qui nessuna delle due componenti orizzontali presenta una spiccata prevalenza sull'altra, né quando la componente verticale è più sviluppata, né quando lo è meno.

- Il 2 gennaio 1949 il fronte freddo, costeggiando la penisola Iberica, si trova rispetto a Roma in una posizione completamente occidentale; le onde microsismi. che non presentano né carattere longitudinale, né carattere trasversale.

- Il 13 agosto 1948 il minimo si trova sul mare del Nord, quindi quasi a Nord rispetto a Roma. Alle 11,00 la componente verticale ha ampiezza piuttosto notevole e la E-W è anche notevole; questa alle 11,02 decresce col decrescere della verticale, ma alle 11,03 mentre la componente verticale rimane ninima, la E.W 
ritorna a registrare ampi microsismi. La N.S rimane sempre notevole. Alle ore 11,05 la componente N-S presenta un minimo nella sua ampiezza, mentre sia la componente verticale che la componente $\mathrm{E} \cdot \mathrm{W}$ presentano una notevole ampiezza. Spesso invece le tre componenti risultano tutte e tre molto ampie o tutte e tre minime. $E$ così pure in vari altri momenti. Alle 19,0l, per esempio, sia la componente verticale che la N-S sono molto sviluppate mentre lo è meno la E-W.

Le onde microsismiche studiate non presentano perciò carattere puro né di onde di Rayleigh (longitudinali con componente verticale) né di onde di Love (trasversali senza componente verticalel. Solo in alcuni momenti assumerebbero carattere di onde di Rayleigh.

Inoltre, scelti alcuni casi chiari di microsismi aventi carattere atlantico, sono stati presi in considerazione tratti del grafico originale per la durata di sei minuti, sia nella componente N-S, che nella componente $\mathrm{E}-\mathbb{W}$.

Dopo averne fatto l'ingrandimento per agevolarne lo studio, è stata calcolata l'ampiezza di ogni massimo (o minimo) di una componente e la corrispondente ampiezza dell'altra. Con queste è stata poi rappresentata la proiezione sul piano orizzontale del vettore spostamento nei punti considerati.

- 115 gennaio 1937 un minimo di pressione è localizzato a $N$ rispetto la stazione registratrice (Trieste). L'onda si comporta dapprima $\left(3^{\mathrm{i}} 01^{\mathrm{m}} 0 \mathrm{9}^{\mathrm{s}} \cdot 3^{\mathrm{h}} 0 \mathrm{1}^{\mathrm{m}} 18^{*}\right)$ come trasversale, dopodiché si dispone invece nella direzione di provenienza; quindi dopo alcuni secondi in cui l'onda non appare né trasversale né longitudinale, per un tratto abbastanza lungo $\left(3^{\ln } 02^{\mathrm{m}} 16^{\mathrm{s}} \cdot 3^{\mathrm{h}} 02^{\mathrm{\prime \prime}} 54,5^{\mathrm{s}}\right)$ si comporta di nuovo come trasversale, per apparire in seguito $\left(3^{\mathrm{h}} 03^{\prime \prime} 39^{\mathrm{s}} \cdot 3^{\mathrm{h}} 04^{\mathrm{m}} 3^{\mathrm{s}}\right)$ come longitudinale. Del resto, tranne alcuni brevissimi tratti in cui l'onda segue la direzione di provenienza o la direzione ad essa ortogonale, essa è situata nella direzione intermedia e perciò non risulta essere né trasversale pura, né longitudinale pura.

- Il 12 gennaio 1937 un minimo è localizzato a NW rispetto a Trieste; in

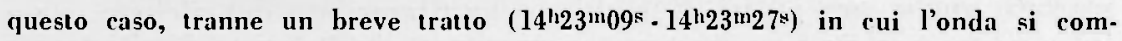
porta come trasversale, essa non ci appare né longitudinale né trasversale pura.

-. Il 16 gennaio 1937 il ciclone è situato a NW di Trieste. Anche in questo caso si tratta prevalentemente di sovrapposizione di onde longitudinali e trasversali; solo nell'intervallo da $7^{1 /} 02^{m} 26^{4}$ a $7^{1} 02^{m} 43^{*}$ l'onda si comporta come longitudinale, da $7^{1} 03^{\prime \prime} 21^{\mathrm{s}}$ a $7^{\mathrm{h}} 03^{\mathrm{m}} 43^{\text {s }}$ come trasversale e sempre come trasversale da $7^{\mathrm{h}} 04^{\mathrm{M}} 11^{\mathrm{s}}$ a $7^{\mathrm{h}} 04^{\mathrm{m}} 29^{\mathrm{N}}$; mentre dopo alcune onde non aventi carattere puro, da $7^{\mathrm{l}} 04^{\mathrm{m}} 45^{\mathrm{s}}$ a $7^{\mathrm{h}} 05^{\mathrm{m}} 02^{*}$ l'onda ci appare come longitudinale.

- Il 21 gennaio 1937 il ciclone è situato a NW di Trieste; anche qui, tranne alcuni brevissimi tratti ( da $4^{\mathrm{h} 59^{m}} 10^{\mathrm{s}}$ a $4^{\mathrm{l}} 59^{\mathrm{m}} 40^{\mathrm{s}}$ l'onda è longitudinale tranne che ai $31^{*}$ e $33^{*}$ ), l'onda non si comporta né come longitudinale né come trasversale.

- Il 22 gennaio 1937 il minimo della pressione è situato sempre a NW rispelto a Trieste. Nei tratti compresi da $4^{\mathrm{h}} 00^{\mathrm{m}} 45^{*}$ a $4^{\mathrm{l}} 01^{\mathrm{m}} 06^{\mathrm{s}}$ e da $4^{\mathrm{h}} 05^{\mathrm{m}} 10^{\mathrm{N}}$ a $4^{\mathrm{l}} 05^{\mathrm{m}} 39^{*}$ l'onda sembra trasversale e da $4^{\mathrm{h}} 02^{\mathrm{m}} \mathrm{H}^{\mathrm{s}} \mathrm{s}$ a $4^{\mathrm{h}} 02^{\mathrm{m}} 55^{\mathrm{N}}$ l'onda appare longitudinale. 
Nel resto del grafico l'onda si comporta come sovrapposizione di onde trasversali e longitudinali con prevalenza però della componente trasversale.

- Il 24 gennaio il minimo della depressione è localizzato a WNW rispetto a Trieste. Anche in questo caso l'onda non si comporta né come longitudinale né come trasversale, bensì come sovrapposizione di ambedue, con una prevalenza però della componente trasversale; l'onda longitudinale si presenta solo nel tratto $14^{11} 02^{\mathrm{m}} 10^{\mathrm{s}} \cdot 14^{\mathrm{l}} 02^{\mathrm{m}} 28^{\mathrm{s}}$.

Da questi esempi risulta quindi che durante quasi tutta la durata del fenomeno il comportamento è anomalo, come cioè se le direzioni di provenienza fossero anche altre, oltre quella principale.

4. Studio del moto dovuto a microsismi. - Vista l'eterogeneità dei risultati di cui sopra, si è pensato di esaminare come si muovono le particelle sotto l'impulso dell'onda microsismica.

Basandomi su un recente studio sulle onde della "fase principale dei terremoti " $\left({ }^{4}\right)$ ho cercato di costruire le proiezioni del moto dovuto ai microsismi, dapprima nel piano orizzontale poi anche nello spazio. Prendendo in considerazione alcuni bei gruppi di onde microsismiche, si sono così ottenute in qualche caso figure pressocché ellittiche, ma in altri casi ciò non è risultato. La particella sollecitata dalle onde microsismiche non descriverebbe perciò sempre delle ellissi nel piano orizzontale, come sembrava risultare da studi precedenti; in alcuni casi quindi lo sfasamento dei moti armonici componenti non è costante. Anche componendo la risultante delle due componenti orizzontali con la componente verticale (registrazioni della stazione di Roma) pochi sono stati i casi in cui la figura risultante è stata ellittica; in questi casi però alcune ellissi ruotano in un senso, altre in senso contrario.

Prendendo in considerazione gli stessi gruppi di onde microsismiche per cui ho tracciato le suddette ellissi, ho studiato il moto della particella tenendo invece conto dell'istante diverso in cui sono da applicare $i$ vettori. Ho ottenuto i grafici nel piano orizzontale e nello spazio di cui alle figg. 3 e 4.

Nel piano orizzontale possiamo subito osservare che dapprima l'onda si comporta come trasversale; quindi, dopo un'onda intermedia avente carattere né longitudinale né trasversale, si comporta per alcune oscillazioni come longitudinale; dopodiché si presenta per alcune oscillazioni come sovrapposizione di onde trasversali e longitudinali.

Nel piano principale (fig. 4) l'andamento è ancora più complicato 

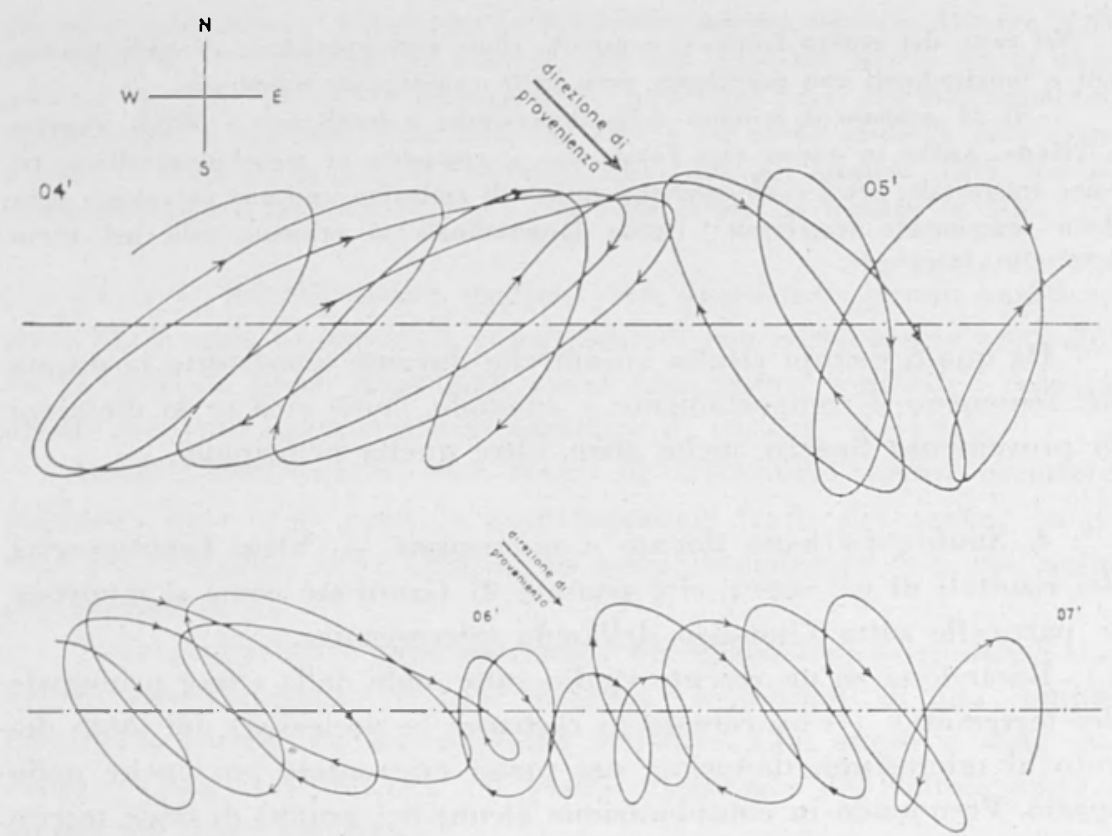

Fig. 3 - Oscillazione sul piano orizzontale in corrispondenza di microsismi (16 gennaio 1937)

pur lasciando intravvedere che il moto principale avviene perpendicolarmente alla direzione di propagazione; ciononostante per l'interferenza degli altri movimenti le particelle si portano lungo una curva ad andamento tortuoso ed irregolare.

5. Conclusioni. - In definitiva, come già sopra si è esposto, $\mathrm{i}$ casi presentati in questo lavoro sono stati scelti in modo da poter dare una risposta chiara sull'eventuale longitudinalità o trasversalità delle onde microsismiche. Invece anche questi casi non danno che risultati ambigui.

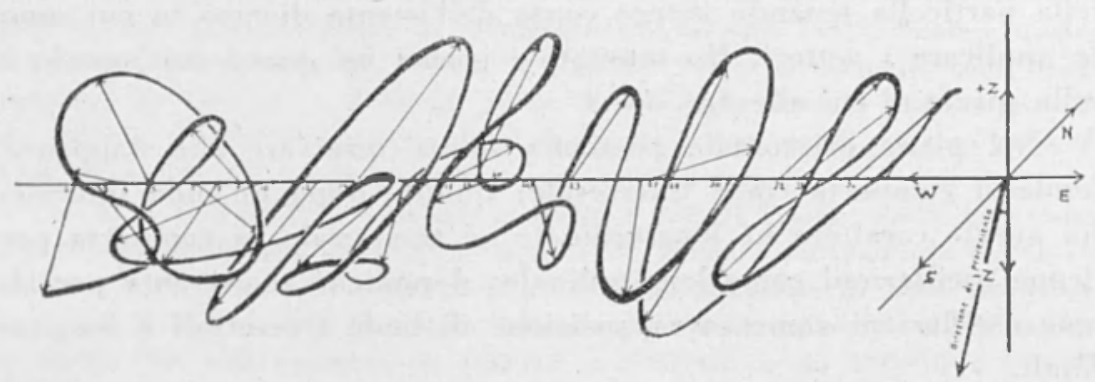

Fig. 4 - Proiezione nel piano principale del moto dovuto a microsismi (13 agosto 1948) 
Non si può quindi affermare che le onde microsismiche si propaghino solo longitudinalmente o solo trasversalmente.

La complessità del movimento registrato in una stazione in corrispondenza ai microsismi si può forse spiegare con l'ipotesi che ora esponiamo. Ammettiamo che fra il centro che dà origine alla perturbazione e la stazione registratrice si trovino una o più superfici di discontinuità sufficientemente inclinate e convenientemente orientate in modo da provocare riflessioni o rifrazioni varie delle onde superficiali che l'investono. È evidente allora che alla stazione registratrice arriveranno onde superficiali corrispondenti a queste varie riflessioni e rifrazioni, e che queste onde presenteranno differenze di fasi variabili a seconda delle varie superfici di discontinuità incontrate. Ciò basterelble da solo a spiegare il caratteristico comportamento osservato nel moto di una particella sotto l'azione dei microsismi, il quale, come albbiamo sopra visto, corrisponde a quello delle classiche figure di Lissayous. Se a tutto questo aggiungiamo il fatto, di cui è giustificata l'ammissione a priori, che almeno nei tragitti iniziali su terraferma i microsismi siano costituiti dai due classici tipi di onde superficiali $Q$ ed $R$, ne deriva un'ulteriore spiegazione della complessità osservata. Difatti, per la diveraa velocità di propagazione dei due tipi di onde $Q$ ed $R$ avremo un continuo interferire delle onde $R$ con le onde $Q$ (nel piano orizzontale).

Tutte queste cause ci sembrano più che sufficienti a spiegare $i$ fatti sperimentali che sopra abbiamo messo in evidenza.

$\mathrm{Ne}$ risulterebbe l'impossibilità di ricavare dalle registrazioni microsismiche di una sola stazione la loro direzione di provenienza con un metodo esclusivamente fisico. Ciò non toglie che essa si possa arguire dal periodo, dall'ampiezza e soprattutto dalla forma dei microsismi che, come sappiamo ${ }^{1}{ }^{1}$, sono caratteristiche delle diverse zone in cui si trova la perturbazione che li genera.

Istituto Nazionale di Geofisica - Osserv. di Trieste - Nov. 1949.

\section{RIASSUNTO}

Scopo di questa pubblicazione era di vedere se dallo studio dei sismogrammi registrati sulle componenti di una sola stazione, si potesse dedurre il moto trasversale o longitudinale dell'onda microsismica, e da questo la direzione di provenienza. É risultato che il compor- 
tamento di detta onda è anomalo e questa anomalia è probabilmente dovuta a fenomeni di riflessioni e rifrazioni successive su superfici di discontinuità nella crosta terrestre.

\section{BIBLIOGRAFIA}

(1) Morelli C.: Contributo allo studio dei microsismi. Annali di Geofisica, I, 4, 621-652, Roma 1948.

(2) D’Henry G.- Morelli C.: Sulle cause dei microsismi. Annali di Geofisica, II, 281-289, Roma 1949.

(3) Bungers R.: Analyse eines mikroseismischen Sturmes. Zeitschrift fïr Geophisik, XVII, 114-135, Gottingen 1941/42.

(4) Menis S. - Morelli C.: Contributo allo studio della cosidetta fase principale di un sismogramma. Annali di Geofisica, II, 545-575, Roma 1949. 